\title{
Tissues in Motion: the Dynamic Environment for Cardiac Morphogenesis
}

\author{
Aleksandrova, Anastasiia ${ }^{1}$; Czirok, Andras ${ }^{1}$; Lansford, Rusty ${ }^{2}$; Rongish, Brenda ${ }^{1}$. \\ ${ }^{1}$ Department of Anatomy and Cell Biology, University of Kansas Medical Center, 3901 Rainbow Blvd. \\ Kansas City, KS 66160, USA \\ ${ }^{2}$ Department of Radiology, Children's Hospital Los Angeles, 4661 Sunset Blvd., Los Angeles, CA \\ 90027, USA
}

During avian heart morphogenesis, transformation of relatively planar cardiac progenitor fields into a multilayered tubular midline heart involves a series of cellular and tissue movements in three dimensions - over a 48 hour period of time. Formation of the heart tube occurs concomitantly with elongation of the foregut (endodermal-lined), and these two developing structures are physically connected via a mesentery.

A gap exists in our understanding of how these large scale, tissue-level events proceed, and the driving forces behind these movements are not fully understood. In this work, our goal was to determine how cardiac precursor cells moved to the midline and if the endoderm (inner layer of foregut) plays a role in this process. We investigated the motion of fluorescently tagged (cardiac myosin light chain 2/CMLC2 transfected) myocardial progenitors in live quail embryos using widefield time-lapse imaging and custom imaging code [1]. Image acquisition was performed on a Leica DMRXA2 or DM6000B automated upright microscope. Using this approach, a selected number of xy fields are imaged at multiple $\mathrm{z}$ steps, with custom software used for subsequent image processing and mosaic formation. Manual tracing of selected image details was performed using custom software [2]. The fibronectin ECM environment for myocardial movement was visualized in vivo with microinjected fluorescent B3D6 antibodies (DSHB, Iowa City, IA), while the nuclei of endocardial progenitors were labeled with transgenic expression of Tie1::H2B-YFP $[3,4]$. The imaging period encompassed the motion of myocardial progenitors from primary heart field(s) to the midline and continued through early heart looping stages. We determined the relative contribution of directed cell autonomous motility (relative to the ECM) versus convective tissue movements (cells and ECM moving in a coordinated fashion) in the assembly of a myocardial layer of the cardiac tube using object tracing and particle image velocimetry/PIV $[5,6]$. Briefly, through an iterative cross-correlation process, this approach results in a high resolution displacement map $[4,5,6]$.

Next, a series of microincision studies that disrupted endodermal folding (regression of the anterior intestinal portal/AIP) were undertaken. Previous work has shown that the endoderm plays a role (signaling and/or mechanical) in midline movements of the myocardial primordia. Varner et al. also indicated that endoderm contraction played a role in the convergence of the primary heart fields [7]. Thus, to dissect the role of the endoderm in myocardial morphogenesis, we labeled endodermal cells with MitoTracker dye (exposure of embryo's ventral side to 350-500 nM dye solution) for time-lapse imaging and incisions were performed in multiple areas through the endoderm and adjacent splanchnic mesoderm of Hamburger-Hamilton $(\mathrm{HH})$ stage 8 embryos, using tungsten needles. Effects on heart morphogenesis and regression of the AIP were analyzed. Next, computational modeling (simulations) were performed to mimic the incisions in silico, and to test model assumptions regarding the role of myocardial and endocardial field contraction. In these models, cell associations are represented as elastic beams, connecting adjacent particles. These beams can be compressed, stretched, bent and twisted. 
Finally, we sought to determine if endodermal and myocardial deformations involve the same molecular mechanisms. Using pharmacological inhibitors, we compared the sensitivity of endodermal and myocardial tissue contractility to perturbation of the actin cytoskeleton (Cytochalasin D, Sigma), Rho kinase (ROCK) signaling (Y27632, Sigma) and focal adhesion kinase function (PF 573228, Tocris Bioscience) in normal and incised embryos. The speed of AIP regression/foregut elongation was used to measure the effects of the reagents on contractility of the endoderm. In order to measure myocardial deformation, CMLC2::GFP+ transfected myocardial progenitors movements were traced in control vs. incised embryos in the vicinity of the incision (wound) edge.

The quantitative data on cardiac precursor cell movements indicated the contribution of cell autonomous motility displayed by myocardial progenitors is limited, albeit slightly higher than that exhibited by endocardial cells. Instead, convective tissue movements play a major role in the arrival of both myocardial and endocardial progenitors at the midline. Time-lapse imaging of embryos in which the myocardial and endodermal cells were both labeled demonstrated that myocardial progenitor fields underwent a series of coordinated deformations that were able to propel their displacements in the anterior direction, relative to the endoderm. In cases where incisions were placed within the myocardial field, parallel or perpendicular to the AIP, the myocardial tissue underwent ventral bending. Computational modeling confirmed that folding of the myocardial sheet in a ventral direction in combination with its contraction along the medial edge reproduced the behavior of myocardial progenitor fields during normal cardiogenesis. Interestingly, myocardial sheet bending occurs concomitantly with its transition to a multi (two or three) layered structure at HH8-9. Finally, we noted that although foregut elongation and myocardial bending are both delayed following perturbation of actomyosin contractility or FAK activity - AIP regression is more dependent on actomyosin contractility than myocardial deformation, while the latter is most dependent on FAK activation. Somewhat surprisingly, we found that expressing a constitutively active isoform of Rho in myocardial cells resulted in a slight decrease in both foregut elongation and myocardial bending, suggesting that myocardial cells may also contribute to foregut elongation.

Our work indicates that, contrary to other published work, the medial movement of myocardial progenitors is not a migration relative to their local ECM environment. We conclude that anteromedially directed movement of cardiac progenitor fields to the site of tubular heart assembly relies on a coordinated influence from: 1) endoderm folding and foregut elongation that drive the convergence at the midline, and 2) deformations intrinsic to the myocardial primordia that mediate apparent movement in the anterior direction, and reposition the myocardia along the dorso-ventral embryonic axis, to achieve the optimal orientation for their fusion at the midline.

[1] A Czirók, PA Rupp, BJ Rongish, et al. J. Microsc. 206 (2002), p 209.

[2] A Czirók, BJ Rongish, CD Little. Dev. Biol. 268 (2004), p. 111.

[3] Y Sato, G Poynter, D Huss, et al. PLoS One 5 (2010), p. e12674.

[4] A Aleksandrova, A Czirok, A Szabo, et al. Developmental Biology 363 (2012), p. 348.

[5] EA Zamir, A Czirók, BJ Rongish, et al. Ann. Biomed. Eng. 33 (2005), p. 854.

[6] EA Zamir, A Czirók, C Cui, et al. Proc. Natl. Acad. Sci. U. S. A. 103 (2006), p. 19806.

[7] VD Varner and LA Taber. Development 139 (2012), p. 1680.

[8] The authors acknowledge funding from NHLBI, HL085694 (BJR); the Mathers Foundation (BJR, AC); and the American Heart Association, AHA predoctoral award (AA). 